\title{
General Discussion on Chemical Abundances
}

Jim Felten: I am worried about abundances and what we mean by different symbols. The notation $X$ usually denotes the fraction of total baryonic mass which is in the form of hydrogen, by which we mean its global value, or largescale average. The decrement $\Delta X$, conventionally written as a positive, rather than negative fractional amount, denotes the global mass fraction which has burned $\mathrm{H}$ into heavier elements at times after the Big Bang - i.e., essentially in stars. This is necessarily less than the mass fraction which has gone into stars. Some $\mathrm{H}$ is expelled from stars; this has been "processed through stars" but not burned. Other $\mathrm{H}$ is locked up unburned in low-mass stars. However $\Delta X$ is defined, the estimates of $\Delta X$ heard at this meeting have been discrepant.

Earlier in the meeting Bernard Pagel said he might be able to set $\Delta X$ as high as 4 or $5 \%$, but he was struggling a bit to do that. Yesterday, Mushotzky told us that the metallicity, $Z$, in rich clusters (closed systems) is about 0.4 of the solar value, and he quoted Cowie as saying that this requires $\Delta X \sim 4 \%$. Next, Madau told us that, to produce minimal background light, he needs $\Delta X \sim 7 \%$ to $16 \%$, with the larger end of this range favored, if a "Madau curve" (burning at high $z$, not $z=0$ ) is used. And Madau said this results in $Z \sim 0.25$. It seems to me that these various estimates of $\Delta X$ are not consistent.

I am asking the experts: What is our consensus on $\Delta X$ ?

Bernard Pagel: I would estimate $\Delta X$ from known stars to be

$$
0.1\left[(\text { white dwarfs }=0.1)+Z_{\odot}+Y_{\odot}-Y_{p}\right]=0.016,
$$

where the last figure refers to primordial helium. $\Delta X$ from the IGM, LSB might be estimated at

$$
\left[Z_{\odot}+Y_{\odot}-Y_{p}\right] / 3 \sim 0.02 .
$$

These add up to a total of $\sim 0.036$.

Ned Wright: All the CIRB measurements are $\sim 4 \sigma$, so a two-fold excess is only $2 \sigma$. However, the Pei \& Fall models are also two times higher, and those are based on making the observed metals.

Martin Harwit: As far as the low-mass stars are concerned, I calculated last year (1999, ApJ, 510, L83) that stars less massive than the Sun could have been shining forever without contributing much to the background radiation. Nor would they add to the metallicity as long as they stay on the Main Sequence. 